\title{
Prevention of Atrophic Nonunion Development by Recombinant Human Bone Morphogenetic Protein-7
}

\author{
Takeshi Makino, David J. Hak, Scott J. Hazelwood, Shane Curtiss, A. Hani Reddi
}

\section{University of California Davis}

Severe periosteal and soft tissue disruption at the time of fracture may result in the formation of an atrophic nonunion. We have developed a reproducible atrophic nonunion in an animal model. The purpose of this study was to evaluate whether the immediate application of recombinant human BMP-7 to the fracture site could rescue the healing process in this nonunion model. A total of 56 three month old Fisher 344 rats were utilized. A $1.25 \mathrm{~mm}$ diameter K-wire was inserted into the femur in a retrograde fashion, and a mid-diaphyseal closed transverse fracture was created using a standard three point bending device. To create a nonunion, the fracture site was exposed and $2 \mathrm{~mm}$ of the periosteum was cauterized on each side of the fracture. The fracture site was immediately treated with either the application of rhBMP-7 $50 \mu$ in $25 \mu$ l of rat tail tendon collagen buffer (BMP-7 group), or with $25 \mu \mathrm{l}$ of rat tail tendon collagen buffer only(Control group). Fracture healing was evaluated with serial radiographs every two weeks for an eight weeks period. Specimens at four and eight weeks were subjected to biomechanical and histological evaluation. None of the Control group healed throughout the eight weeks experimental duration. At four weeks $63 \%$ of the BMP-7 group had healed, and all had healed by six weeks. This investigation showed pronounced differences between the BMP-7 group and the Control group both histologically and biomechanically. In conclusion, we have demonstrated that the immediate application of BMP-7 may rescue the fracture healing process and prevent the development of nonunion following severe periosteal disruption.

\section{Introduction}

Clinical and experimental evidence of healed frac-tures has highlighted the importance of minimizing periosteal disruption at the fracture site [5,15,17,20, 21,25]. During an initial stage of fracture healing, the periosteal cells first differentiate into osteoblasts fol-lowed by intramembranous ossification [3]. Severe periosteal and soft tissue disruption at the time of fracture may result in atrophic nonunion. We have devel-oped a reproducible nonunion model in the rat femur that simulates the clinical condition in which there is periosteal disruption without segmental bone defect, and showed pronounced differences between the experi-mental nonunions and the standard closed fractures both histologically and biomechanically [14].

Bone morphogenetic proteins (BMPs) govern the key steps in new bone formation $[23,24]$. BMP-7 has been shown to act on the proliferation and differentiation of osteoprogenitor cells into bone forming cells [2]. The therapeutic potential of recombinant 
human BMP-7 (rhBMP-7) has been examined in animal models of critical-sized segmental bone defects, osteochondral defects, spinal fusion, and distraction osteogenesis $[8,16,18,19]$. Some clinical studies have also been performed on tibia1 nonunions and fibular defects [lo, 111. The purpose of this study was to evaluate whether immediate application of rhBMP-7 to the fracture site could rescue the healing process and prevent nonunion formation following severe periosteal disruption.

\section{Materials and methods}

\section{Experimental design}

Fifty-six male 3 months old Fisher 344 rats were included in this study. Their mean and standard deviation of body weight was $298 \mathrm{~g}$ (standard deviation $\pm 14 \mathrm{~g}$ ). All surgical procedures were performed under general anesthesia and normal sterile conditions. Anesthesia was performed with 4\% Halothane inhalation, followed by Ketamine hydrochloride $(80 \mathrm{mg} / \mathrm{kg})$ and Xylazine hydrochloride $(8 \mathrm{mg} / \mathrm{kg})$ administered intraperitoneally.

A lateral parapatellar knee incision on the right limb was made to expose the distal femoral condyle. A $1.25 \mathrm{~mm}$ diameter K-wire was inserted from the trochlear groove into the femoral canal in a retrograde fashion with use of a motor-driven drill. The wire was advanced through the greater trochanter and out of the skin until its distal end was positioned deep to the articular surface of the knee. A $5 \mathrm{~mm}$ incision in the skin was made around the K-wire and the wire was then cut close to the proximal femur. After irrigation with sterile saline, the wounds were closed with 5-0 nylon suture. A closed transverse femoral shaft fracture was then created in the right femur of each rat using a three point bending apparatus with a drop weight (R. Zarb, State University New York, Brooklyn, NY, USA) following the method of Bonnarens and Einhorn [4]. Following this procedure, all of the rats received additional surgery to create a nonunion in the fractured shaft. In order to produce the nonunion, the fractured site was exposed through a lateral approach, and the periosteum was cauterized (Loop tip surgical cautery, Abco Dealers Inc., Nashville, TN, USA) circumferentially for a distance of $2 \mathrm{~mm}$ on each side of the fracture. The muscle was protected to preserve all soft tissue except the periosteum around the fracture site. The wound was then irrigated with $10 \mathrm{cc}$ of sterile saline and the wound was dried with gauze.

Radiographs were obtained immediately post-operatively to confirm fracture configuration and K-wire placement. Four animals had comminuted fractures and one developed an infection. These animals were eliminated from the study and replaced. All of the remaining fractures were mid-diaphyseal transverse fractures. With the exception of 
the animal with the infection all of the wounds healed uneventfully. No increase in local swelling was observed in the BMP treated animals.

Animals were randomized into two groups of 28. One group was treated with rhBMP-7 $50 \mathrm{pg}$ in $25 \mathrm{pl}$ of rat tail tendon collagen buffer (BMP-7 group), and the other group was treated with $25 \mathrm{pl}$ of rat tail tendon collagen buffer only (Control group). The concentration of rat tail tendon collagen buffer was $0.1 \mathrm{Smglml}$ in $50 \mathrm{mM}$ acetic acid. The rhBMP-7 or the collagen buffer alone was carefully applied anteriorly, laterally, and posteriorly to the experimental nonunion site with a pipet. The viscosity of the collagen buffer prevented extravasation into the surrounding soft tissues. The muscle fascia and skin were closed in layers with 5-0 nylon sutures. Post-operative pain was managed by administration of subcutaneous injection of buprenorphine hydrochloride after surgery. The rats were fed a standard maintenance diet and provided water ad libitum. Unprotected weight bearing was allowed immediately post-operatively.

Four animals from each group were euthanized with an excess of carbon dioxide gas at two weeks and underwent histological evaluation. Twelve animals from each group were maintained for intervals of 4 and 8 weeks. Eight specimens from each time point were randomly selected for biomechanical testing as described below at 4 and 8 weeks. The four remaining specimens from each group were processed for histological study. This research protocol was approved by the Institutional Animal Care and Use Committee, following all appropriate guidelines.

\section{Radiological evaluation}

The progress of bone formation was monitored on radiographs (model 43855A; Faxitron X-ray, Buffalo Grove, IL, USA). Radiographs of all rats were obtained following immediately after surgery and at 2 week intervals until the time of sacrifice. This was done under anesthesia with the animal prone and both limbs fully abducted and external rotated. Radiographs of each animal were assessed by three blinded independent observers to judge whether the fractures united or not. Fracture union was determined by the presence of a bridging callus on two cortices. Concordance of the three observers was required. When the reviewers differed in their interpretation the classification of healed versus nonhealed was made by group consensus.

\section{Histological evaluation}


Histological evaluation was performed on four animals from each group at 2, 4 and 8 weeks after surgery. Right femurs were harvested and fixed in $4 \%$ paraformaldehyde in $0.1 \mathrm{M}$ phosphate buffer for $24 \mathrm{~h}$ at $4^{\circ} \mathrm{C}$, defatted in $70 \%$ ethanol, decalcified with $10 \%$ formic acid in citrate for 4 days at $4^{\circ} \mathrm{C}$ and embedded in paraffin. Paraffin sections $4 \mathrm{pm}$ thick were cut and stained with toluidine blue for histological evaluation. The degree of fracture healing was evaluated using a five point scale proposed by Allen et al. [I] According to this classification system grade four represents complete bony union, grade three represents an incomplete bony union (presence of a small amount of cartilage in the callus), grade two represents a complete cartilaginous union (well-formed plate of hyaline cartilage uniting the fragments), grade one represents an incomplete cartilaginous union (retention of fibrous elements in the cartilaginous plate), and grade zero indicates the formation of a pseudoarthrosis (most severe form of arrest in fracture repair).

\section{Biomechanical evaluation}

Biomechanical evaluation was performed on eight animals from each group at 4 and 8 weeks. Following euthanasia the right femurs and the contralateral nonfractured intact femurs were harvested and dissected free of surrounding muscles. After removal of the intramedullary K-wires, the ends of both femurs were imbedded in Wood's metal (Alfa Aesar, Ward Hill, MA, USA). Specimen length was statistically similar between the BMP-7 group $(17.5 \pm 1.9 \mathrm{~mm})$ and the Control group $(17.2 \pm 1.7 \mathrm{~mm})(p,>0.05)$. The specimens were mounted in a Frankel-Burstein axial torsion-testing machine [6] that was modified to operate under computer control. The standard swinging-pendulum mechanism was replaced with an electronic stepper motor drive (model 083062-1-8-03 1-010, Parker Compumotor, Rohnert Park, CA, USA). Rotational displacement was measured with a precision potentiometer (model 793341-14092, Gould. Inc., Cleveland, OH, USA), and torque was measured with a torque cell (model 2105, Eaton Corporation, Troy, MI, USA). Specimens were tested in torsion at a rate of $50^{\circ}$ per minute until failure or through an arc of $45^{\circ}$. Rotational displacement and torque data were collected at $60 \mathrm{~Hz}$ using a digital data acquisition system (model K500A, Keithley Instruments, Cleveland, OH, USA). Maximum torque to failure was measured directly from the data and torsional stiffness was calculated from a regression of the linear portion of the torque versus angular displacement curve. The energy absorption to maximum torque was calculated as the area under the curve to the maximum value. The contralateral intact femur was also tested to calculate normalized values of maximum torque to failure (percent maximum torque), stiffness (percent stiffness) and energy absorption to maximum torque (percent energy) in order to reduce the influence of individual animal differences. 


\section{Statistical analysis}

A two factor analysis of variance (ANOVA) was performed on the values of the biomechanical properties. The factors were the time point and the experimental model (BMP-7 group, Control group and Intact group). Second, ANOVA was performed on the normalized data of the biomechanical properties (BMP-7 group and Control group) to account for individual animal differences. Significance was defined as p values less than 0.05. When appropriate, a Bonferroni-Dunn post hoc test was performed to determine differences between the models at the various time points. For the post hoc test, the level of signifi-cance was adjusted to 0.0018 to account for multiple comparisons.

\section{Results}

\section{Radiological evaluation}

Radiographs taken just after surgery showed trans-verse mid-diaphyseal femoral fractures in both the BMP-7 group and the Control group (Fig. 1A, E). The numbers of radiographic fracture unions during each period were shown in Table 1. Sixty-three percent of the BMP-7 group healed at 4 weeks, and they all healed at 6 weeks after surgery. In contrast, bridging callus was not seen in the Control group, even at 8 weeks after surgery, with 10 of 12 specimens classified as atrophic nonunions based on their radiographic appearance (Fig. $1 \mathrm{H}$ ). The radiographic findings were inconclusive in two of the Control group, but their bio-mechanical properties confirmed the development of a nonunion. The BMP-7 group displayed abundant callus around the fracture sites at two weeks (Fig. 1B). At 4 weeks, most of the callus bridged between both ends of the fractured bones and the cortical gaps disappeared (Fig. IC). After forming a bridging callus, bone remod-eling and consolidation began (Fig. 1 D). No evidence of heterotopic ossification was observed in the surrounding soft tissues of any of the BMP-7 treated specimens. On the other hand, the Control group did not have much callus fonnation around the fracture sites. Some callus formed along the periosteum away from the fracture site, but this never extended to bridge the fracture site (Fig. 1 F, G). The ends of the fractured bone became round and were resorbed, appearing to be established nonunions at 8 weeks (Fig. 1H).

The largest area of callus was observed posterior to the femur. The collagen and BMP was applied uni-formly to the anterior, lateral and posterior aspect of the fracture. Although extravasation of the material was not seen acutely, this larger area of posterior callus may represent gradual flow of BMP and collagen into the dependent region following the surgical procedure. 
At 2 weeks, the BMP-7 group displayed intramem-branous ossification in the periosteal tissue and newly formed trabecular bone at the fracture site (Fig. 2A). Newly formed trabecular bone was predominant corn-ponent of this thick callus. At 2 weeks, the Control group exhibited chondrocytes and nonbridging endo-chondral ossification (Fig. 2D), but there was no bone formation on the site of periosteal cauterization. At four weeks after surgery, the callus in the BMP-7 group had united and the chondrogenic areas had disappeared (Fig. 2B). The fractured bone was covered with newly formed trabecular bone and achieved bony union. In contrast, a large gap persisted between the surfaces of woven bone in the Control group at four weeks (Fig. 2E). At eight weeks, the united bone in the BMP-7 group had remodeled with progressive decrease in the thickness of the woven bone (Fig. 2C). In comparison, at 8 weeks in the Control group, the fibrous tissue was interposed at the fracture site and the ends of the frac-tured bone became round and resorbed (Fig. 2F). This is consistent with the histological finding of atrophic nonunions.

In the Control group, histological grading of the frac-ture healing was similar among all specimens within each time-point. Histology of controls was graded as 1 at week 2 , and 0 at weeks 4 and 8 . In the BMP-7 treated specimens the mean histology grade was 1.5 at week 2 , and 4 at weeks 4 and 8 .

\section{Biomechanical evaluation}

The means and standard deviations of the maximum torque to failure, the stiffness and the energy absorp-tion to maximum torque are shown in Table 2. The maximum torque, stiffness and energy absorption values were normalized by the respective values from the intact femurs (percent maximum torque, percent stiffness and percent energy) and average values were calculated for each group (Fig. 3). Significant increase in all the mechanical properties was seen in the BMP-7 group compared to the Control group at both 4 and 8 weeks. No significant difference in all the mechanical properties was seen between the BMP-7 group and the Intact group at both 4 and 8 weeks. All the mechanical properties of the BMP-7 group gradually increased between at 4 and 8 weeks, but no significant differences between 4 and 8 weeks was seen in all the mechanical properties. The mean values of percent maximum torque at 8 weeks and percent energy at 4 and 8 weeks of the BMP-7 group exceeded 100\% (Fig. 3A, C). All the mechanical properties of the Control group were less than $25 \%$ of their corresponding intact values at 4 and 8 weeks (Fig. 3).

\section{Discussion}

Most previous studies have evaluated the effect of BMP treatment in criticalsized segmental defects $[8,13,26]$. However, these models may not adequately simulate 
the biological and mechanical environment that clinically leads to nonunion development. Our model was specifically developed to better simulate the altered biological and mechanical environment that is seen in high energy fractures. In high energy fractures, there is often significant disruption of periosteum and surrounding soft tissues [12]. Clinical and experimental evidence indicates that periosteal disruption at the frac-ture site impairs fracture healing $[5,15,17]$.

The present study demonstrated that immediate application of rhBMP-7 into the fracture site enhances the fracture healing process of this nonunion model in radiographic, histological and biomechanical properties. Serial radiographs indicated that $63 \%$ of the BMP-7 treated group united radiographically at 4 weeks and at 6 weeks all displayed bridging callus indicating a uni-ted fracture. In contrast, the cauterized nonunion model did not heal by 8 weeks after fracture. Our radiograph-ical findings of the BMP-7 treated group at 2 weeks revealed a large amount of callus. Histologically this callus already consisted mainly of newly formed trabecular bone, with few small chondrogenic areas. In contrast, a nonbridging cartilaginous callus was present in the nonunion group at 2 weeks. It is known that BMPs act at an early stage of bone induction. They induce, promote and maintain both bone and cartilage formation [22]. Recently it has been reported single injection of high dose rhBMP-2 into the standard rat fracture model induced a large amount of fracture callus and enhanced fracture healing [9]. Significant periosteal and soft tissue disruption occurs in open tibia fractures, which are often complicated by delayed union and nonunion. Serial debridement of open fractures may cause additional periosteal damage and further resection of necrotic or contaminated tissues may be required. Application of rhBMP-2 (1.5 $\mathrm{mg} / \mathrm{ml}$ ) at the time of open fracture wound closure has been shown to accelerate fracture healing and reduce the frequency and invasive-ness of secondary interventions to achieve union in hu-mans [12]. Similarly in our study, the application of BMP appears to accelerate endochondoral bone formation, rapidly converts cartilaginous callus to bone, even in the presence of severe periosteal disruption. In contrast, in the absence of supplemental BMP there is resorption of the fracture ends with interposition of the fibrous tissue.

Biomechanical torsional testing revealed that percent maximum torque was six times larger, percent stiffness was eleven times larger, and percent energy was ten times larger for the BMP-7 treated group compared to the nonunions at 8 weeks. Each of these mechanical properties had statistically significant differences between the BMP-7 treated group and nonunion group. The nonunions did not have the remodeling callus around the fracture site and the two fractured fragments were connected with only fibrous tissue, making the nonunion weaker than the BMP-7 treated group throughout the study. The biomechanical evaluation supported the radiographic and histological findings of the fracture repair by rhBMP-7.

The Control groups used in this study were treated with $25 \mu \mathrm{l}$ of collagen applied 
to the fracture site at the time of cauterization. The mechanical results of these collagen treated controls are comparable to the results seen in our previously reported study in which nothing was applied following cauterization [14]. In that study, the mean maximum torque to failure in animals treated with cauterization alone was $0.052 \mathrm{Nm}$ at 4 weeks, and $0.088 \mathrm{Nm}$ at 8 weeks. This indicates that the enhanced bone healing seen in this study was due to the rhBMP-7 rather than the collagen carrier.

A dose response study was not performed to deter-mine the optimal rhBMP-7 dosage to promote bone formation in this model. The amount selected, 50 .tg, was based on successful union reported in a rat segmen-tal femoral defect model [7]. That study also reported the use of a smaller dose, $11 \mu \mathrm{g}$, however the amount of bone formation was not as great with the lower dose. In a recently published report of percutaneous rhBMP-2 injection in a closed femur fracture model, an even higher dose, $80 \mu \mathrm{g}$, has been used [9].

We have shown the immediate application of rhBMP-7 may rescue the healing process and prevent the development of nonunion. The present study dem-onstrates rhBMP-7 has the potential to accelerate the healing process of the high energy fractures in clinical situations.

\section{Acknowledgement}

This research was supported by a grant from the Orthopaedic Research and Education Foundation. RhBMP-7 was a generous gift from Dr. T. K. Sampath (Creative BioMolecules, Hopkinton, MA, USA).

\section{References}

[1] Allen HL, Wase A, Bean WT. Indomethicin and aspirin: effects of nonsteroidal antiinflammatory agents on the rate of fracture repair in the rat. Acta Orthop Scand I 980;5 1:595-600.

[2] Asahina I, Sampath TK, Nishimura I, Hauschka PV. Human osteo nic protein-i induces both chondroblastic and osteoblastic differentiation of osteoprogenitor cells derived from newborn rat calvaria. J Cell Biol 1993;123:921-33.

[3] Bolander ME. Regulation of fracture repair by growth factors. Proc Soc Exp Biol Med I 992;200: 165-70.

[4] Bonnarens F. Einhorn TA. Production of a standard closed fracture in laboratory animal bone. J Orthop Res 1984;2:97-101.

[5」 Buckwalter JA, Cruess RL. Healing of the musculoskeletal tissues. In: Rockwood CA, Green DP, Bucholz RW, Heckman JD, editors. Fractures in adults. 4th ed. Philadelphia: Lippincott; 1996. 
[6] Burstein AH, Frankel VH. A standard test for laboratory animal bone. J Biomech 197i;4:155-8.

[7] Chen X, Kidder LS, Lew WD. Osteogenic protein-I induced bone formation in an infected segmental defect in the rat femur. J Orthop Res 2002;20: 142-50.

[8] Cook SD, Wolfe MW, Salkeld SL, Rueger DC. Effect of recombinant human osteogenic protein-i on healing of segmental defects in non-human primates. J Bone Joint Surg Am I 995;77- A:73-50.

[9] Einhorn TA, Majeska RJ, Mohaideen A, et al. A single injection of recombinant human bone morphogenetic protein-2 accelerates fracture repair. J Bone Joint Surg Am 2003;85-A:1425-35.

[10] Friedlaender GE, Perry CR, Cole JD, et al. Osteogenic protein-i (bone morphogenetic protein-7) in the treatment of tibial nonunions. J Bone Joint Surg Am 2001;83-A(Suppl l):Sl-8.

[11] Geesink RG, Hoefnagels NH, Bustra SK. Osteogenic activity of OP-I bone morpho即netic protein (BMP-7) in a human fibular defect. J Bone Joint Surg Br i999;81-B:710-8.

[12] Govender 5, Csimma C, Genant HK, et al. Recombinant human bone morphogenetic protein-2 for treatment of open tibial fractures: a prospective, controlled, random Led study of four hundred and fifty patients. J Bone Joint Surg Am 2002;84- A:2 123-34.

[13] Heckman JD. Bone morphogenetic protein but not transforming growth factor-beta enhances bone formation in canine diaphyseal nonunions implanted with a biodegradable composite polymer. J Bone Joint Surg Am 1999;8i:1717-29.

[14] Kokubu T, Hak Di, Hazelwood Si, Reddi AH. Development of an atrophic nonunion model and comparison to a closed healing fracture in rat femur. J Orthop Res 2003;21:503-10.

[15] Landry PS. Effect of soft-tissue trauma on the early periosteal response of bone to injury. J Trauma 2000;48:479-83.

[16] Louwerse RT, Heyligers IC, Klein-Nulend J, et al. Use of recombinant human osteogenic protein-I for the repair of subchondral defects in articular cartilage in goats. J Biomed Mater Res 2000;15:506-16.

[17] McKibbin B. The biology of fracture healing in long bones. J Bone Joint Surg Br 1978;60-B:150:2.

[18] Mermer Mi, Gupta MC, Wheeler DL, et al. Efficacy of osteo-genic protein-l in a challenging multilevel fusion model. Spine 2004;29:249-56.

[19] Mizumoto Y, Moseley T, Drews M, et al. Acceleration of regenerate ossification during distraction osteogenesis with recombinant human bone morphogenetic protein-7. J Bone Joint Surg Am 2003;85-A:S151-8.

[20] Oni 00, Stafford H, Gregg PJ. An experimental study of the patterns of periosteal and endosteai damage in tibial shaft fractures using a rabbit trauma model. J Orthop Trauma i989;3:142-7. 
[21] Park SH, O'Connor K, Sung R, McKellop H, Sarmiento A. Comparison of healing process in open osteotomy model and closed fracture model. J Orthop Trauma 1999;13:i1午20.

[22] Reddi AH. Role of morphogenetic proteins in skeletal tissue engineering and regeneration. Nat Biotechnol 1998; 16:247-52.

[23] Sampath TK, DeSimone DP, Reddi AH. Extracellular bone matrix-derived growth factor. Exp Cell Res 1982;142:460-4.

[24] Sampath TK, Reddi AH. Homology of bone-inductive proteins from human, monkey, bovine, and rat extracellular matrix. Proc Natl Acad Sci USA 1983;80:6591-5.

[25] Whiteside LA. The effects of extraperiosteal and subperiosteal dissection. II. On fracture healing. J Bone Joint Surg Am I 978;60:26-30.

[26] Yasko AW, Lane JM, Fellinger EJ, et al. The healing of segmental bone defects, induced by recombinant human bone morphogenetic protein (rhBMP-2). J Bone Joint Surg Am i992;74-A:659-70. 

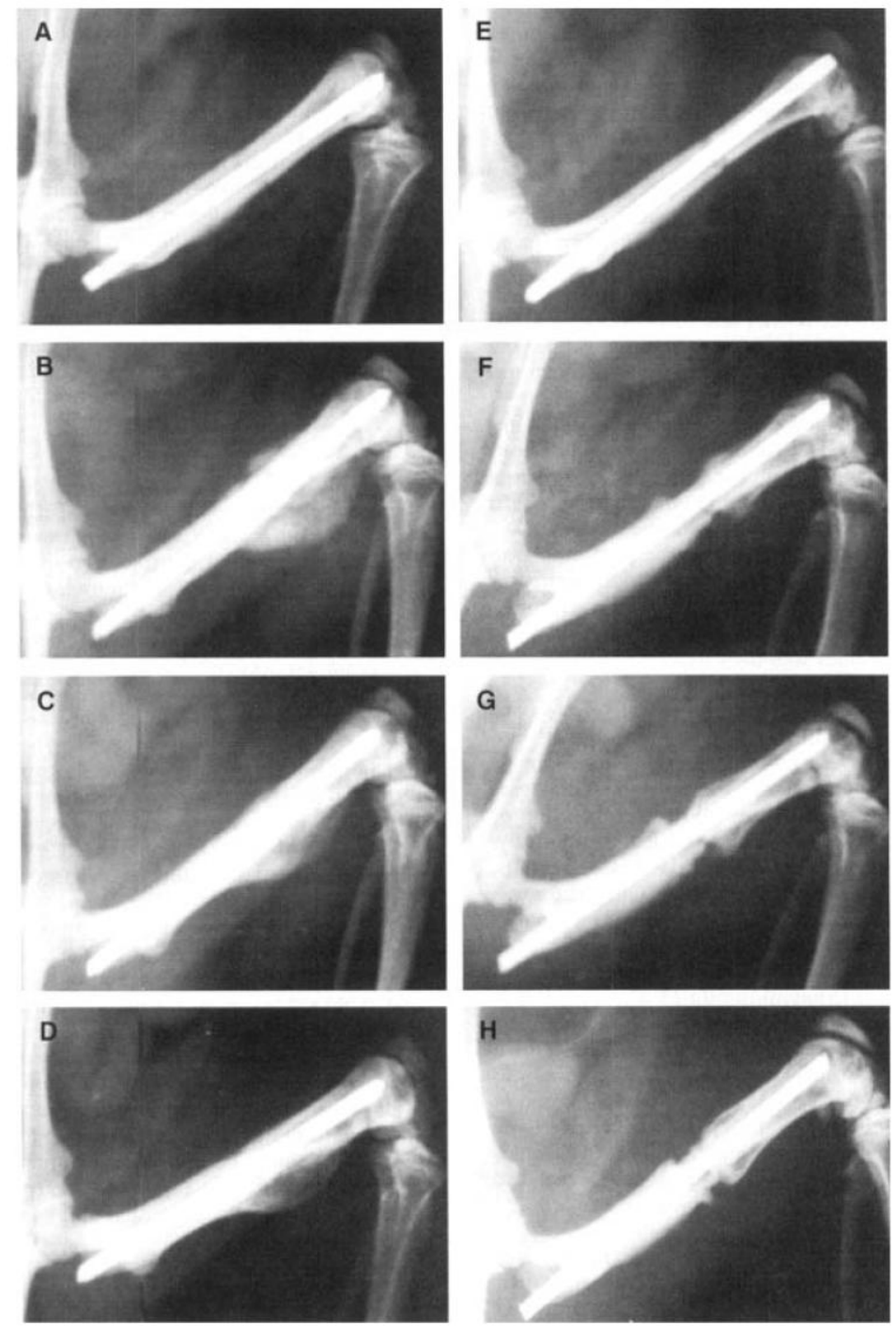

Fig. 1. Radiographs of both the BMP-7 group (A-D) and the Control group (E-H) obtained immediately after surgery (A, E), 2 weeks after surgery (B, F), 4 weeks after surgery (C, G), and 8 weeks after surgery (D, H). Note the union in the BMP-7 group and the gap in the Control group.

Table 1

Serial radiographic evaluation of fracture union

\begin{tabular}{lll}
\hline Period after surgery (weeks) & BMP-7 group & Control group \\
\hline 2 & $0 / 28(0 \%)$ & $0 / 28(0 \%)$ \\
4 & $15 / 24(63 \%)$ & $0 / 24(0 \%)$ \\
6 & $12 / 12(100 \%)$ & $0 / 12(0 \%)$ \\
8 & $12 / 12(100 \%)$ & $0 / 12(0 \%)$ \\
\hline
\end{tabular}



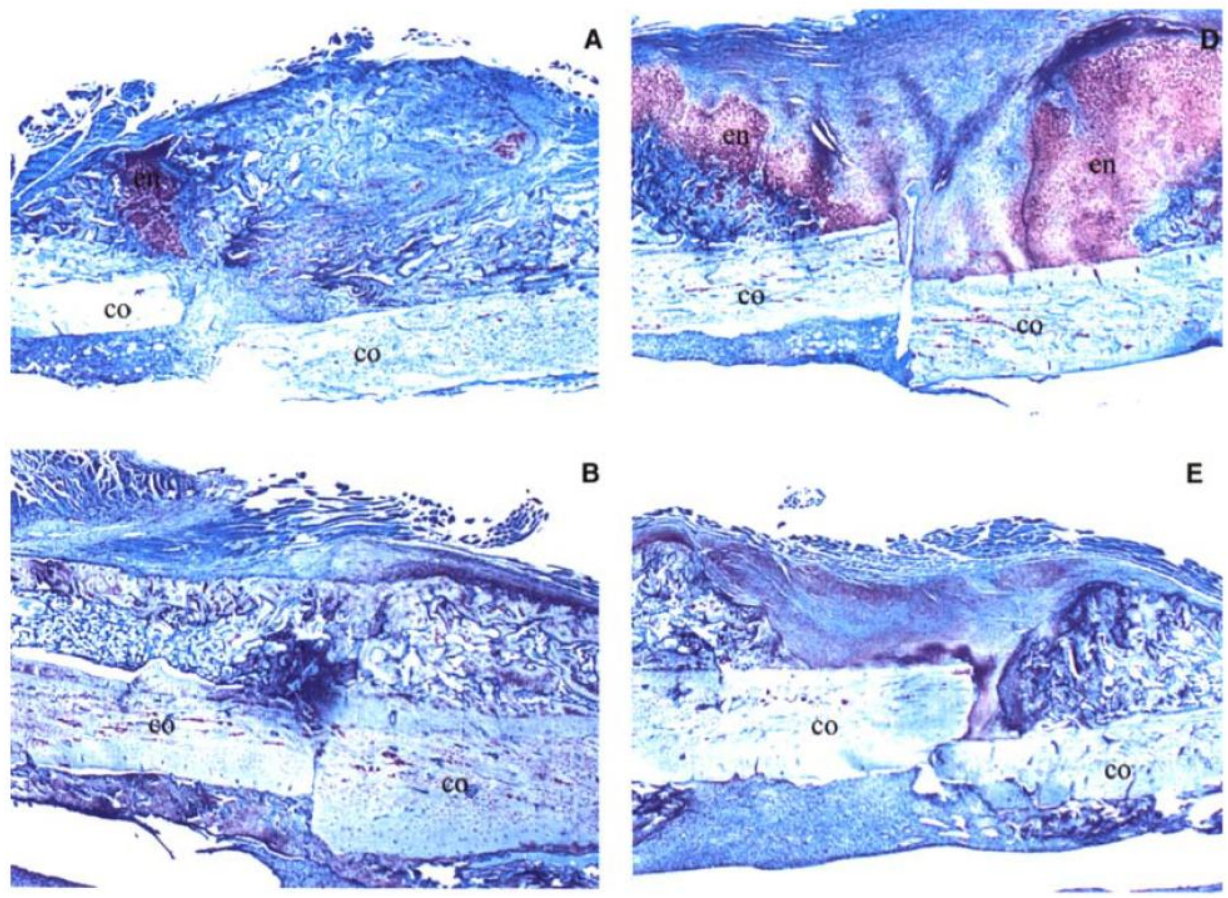

3

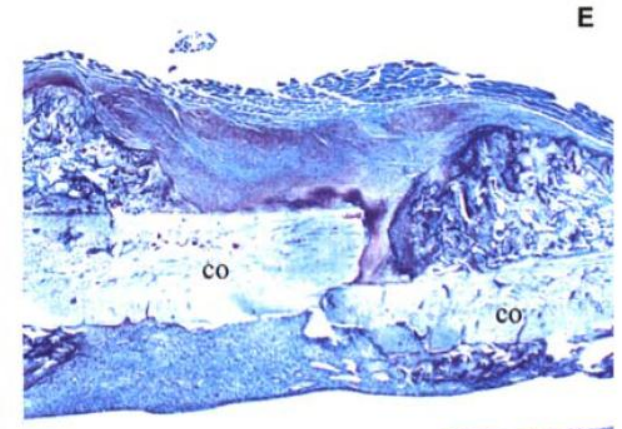

C
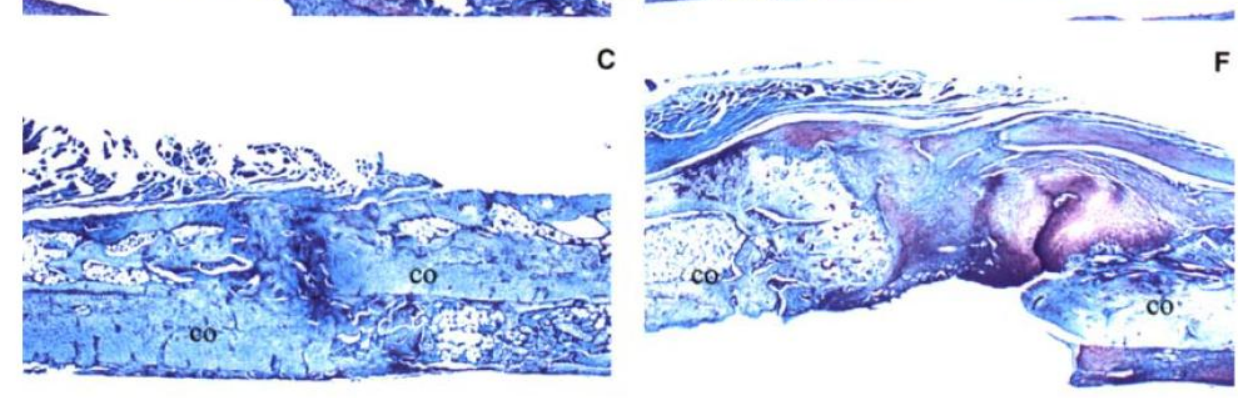

$1 \mathrm{~mm}$

Fig. 2. Histological findings of the BMP-7 group (A-C) and the Control group (D-F). Sections were stained with toluidine blue. The BMP-7 group at 2 weeks displayed newly formed trabecular bone at the fracture site (A). The Control group exhibited chondrocytes and nonbridging endochondral ossification (D). The BMP-7 group united at 4 weeks after surgery (B). The Control group still had a large gap (E). At 8 weeks, the united bone had remodeled (C). No healing was observed and the cortical bone was resorbed (F). en: endochondral ossification. co: cortical bone. Bar, $1 \mathrm{~mm}$.

Table 2

Biomechanical evaluation in BMP-7 group, Control group and Intact group

\begin{tabular}{lccc}
\hline & ${\text { BMP-7 } \text { group }^{\mathrm{a}}}^{\mathrm{a}}$ & Control group $^{\mathrm{a}}$ & Intact $^{\mathrm{a}}$ \\
\hline Maximum torque to failure [Nm] & $0.249 \pm 0.065^{*}$ & & \\
4 weeks & $0.409 \pm 0.108^{*}$ & $0.041 \pm 0.028$ & $0.278 \pm 0.051$ \\
8 weeks & & $0.069 \pm 0.046$ & \\
Stiffness [Nm/rad] & $0.840 \pm 0.284^{*}$ & $0.123 \pm 0.093$ & $1.020 \pm 0.144$ \\
4 weeks & $1.373 \pm 0.336^{*}$ & $0.117 \pm 0.023$ & $1.392 \pm 0.164$ \\
8 weeks & & \\
Energy absorption to maximum torque [Nm rad] & $0.0623 \pm 0.0361^{* *}$ & $0.0065 \pm 0.0044$ & $0.0474 \pm 0.0159$ \\
4 weeks & $0.0830 \pm 0.0417^{*}$ & $0.0078 \pm 0.0062$ & $0.0554 \pm 0.0226$ \\
8 weeks
\end{tabular}

No significant difference of the values between in the BMP-7 group and in the Intact group $(p>0.05)$.

* Significantly greater than the values in the Control group $(p<0.0001)$.

" Significantly greater than the values in the Control group $(p=0.0007)$.

a The values are given as the mean and standard deviation. 

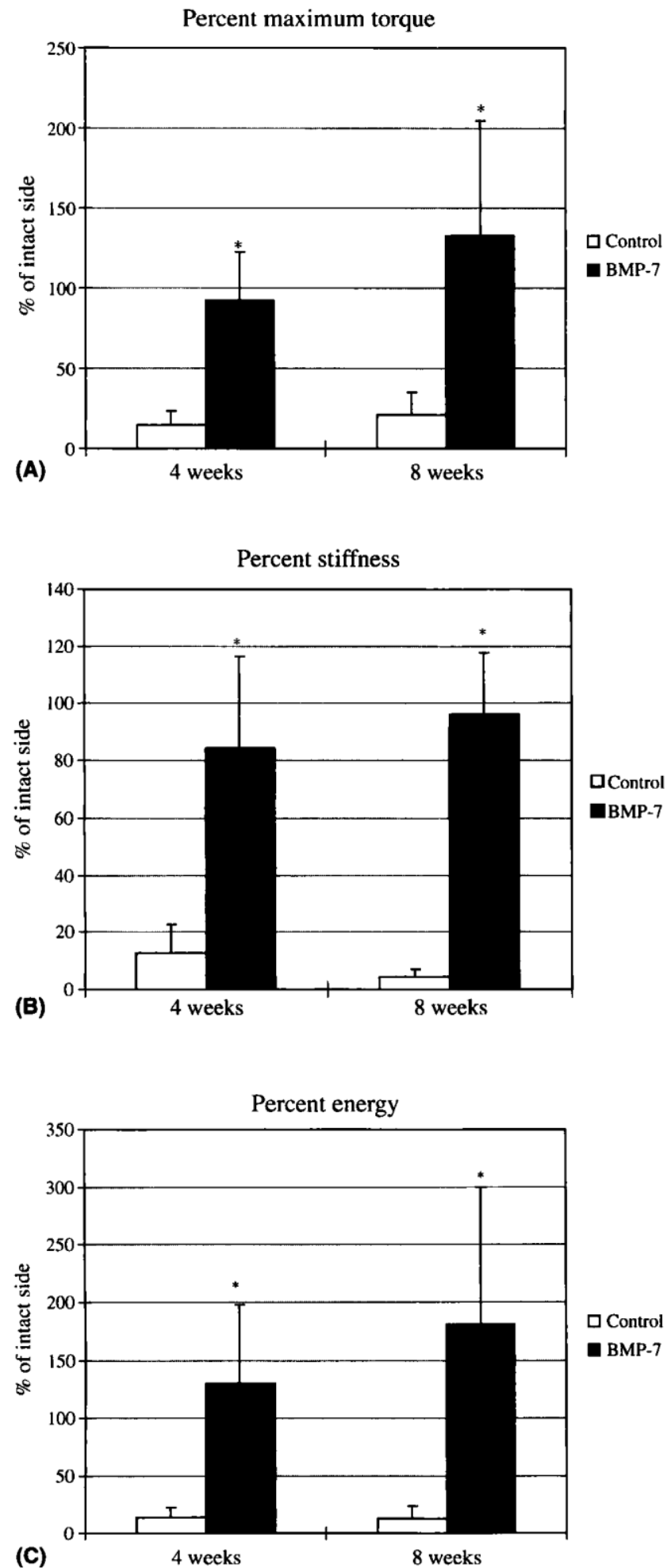

Fig. 3. Biomechanical properties of the BMP-7 group and the Control group normalized by the respective values of the intact femurs. (A) Percent maximum torque (maximum torque to failure of fractured femur/nonfractured intact femur $\times 100$ ). (B) Percent stiffness (stiffness of fractured femur/nonfractured intact femur $\times 100$ ). (C) Percent energy (energy absorption to maximum torque/nonfractured intact femur $\times 100$ ). ${ }^{*}$ Significant differences were found between the Control group and the BMP-7 group in percent maximum torque, percent stiffness, and percent energy measurements $(p<0.0001)$. 